0038-1098(95)00574-9

\title{
Spin-glass-like behaviour of Fe-based Diluted Magnetic Semiconductors
}

\author{
Y.F.Chen \\ Department of Physics, National Taiwan University, Taipei, Taiwan 10617, R.O.C.
}

W.C.Chou

Chung Yuan Christian University, Chung Li, Taiwan 32023, R.O.C.

A.Twardowski

Institute of Experimental Physics, Warsaw University, 00681 Warsaw, Poland

(Received 3 March 1995; received in revised form 5 June 1995 by M. Grynberg)

\begin{abstract}
We measured zero field cooled (ZFC), field cooled (FC) and thermoremanent (TRM) magnetization of Fe-based diluted magnetic semiconductors: $\mathrm{HgFeSe}, \mathrm{CdFeS}, \mathrm{ZnFeSe}$ and $\mathrm{ZnFeS}$. For all these systems, at sufficiently low temperatures, we observed typical spin-glass magnetic behaviour: pronounced difference between ZFC and FC magnetization, as well as nonzero TRM.
\end{abstract}

Diluted magnetic semiconductors (DMS) are materials based on classical II-VI, IV-VI, II-V or III-V semiconductors for which fraction of nonmagnetic cations is substituted by magnetic ions (typically transition metal ions)[1]. From magnetic point of view these materials represent systems of localized magnetic moments (resulting from localized $d$ or $f$ electrons) randomly distributed in nonmagnetic host lattice (fcc or hcp for II-VI and III-V compounds), coupled by long ranged antiferromagnetic (AF) interaction [2]. This interaction yields frustration of magnetic moments, which is believed to be a possible reason for a spin-glass (SG) phase [3] formation for DMS $[2,4]$.

In fact, for the mostly investigated Mn-based DMS a typical spin-glass-like behavior was observed [2,5,6,7]. The fingerprints of this behaviour are [3]: a). a cusp-like (or kink-like) anomaly in a.c. susceptibility (the temperature, at which the anomaly is observed is denoted as "freezing temperature" $T_{f}$ ), b). history dependent d.c. susceptibility (or low field magnetization) for $\mathrm{T}<\mathrm{T}_{\mathrm{f}}$ i.e. the difference between zero field cooled (ZFC) and field cooled (FC) susceptibility, as well as nonvanishing thermoremanent magnetization (TRM) below $\left.T_{f}, c\right)$. monotonous temperature dependence of magnetic specific heat in the vicinity of $T_{f}$ , d). frequency dependence of $\left.T_{f}, e\right)$. nonlinear susceptibility. We notice that the situation for Mn-DMS, although not completely understood, is relatively the simplest, since $\mathrm{Mn}$ ions represent spin-only $(\mathrm{S}=5 / 2, \mathrm{~L}=0)$ magnetic moments $[2,7]$.

For Fe-based DMS the situation is more complicated. Due to nonvanishing orbital momentum of $\mathrm{Fe}^{++}$ions $(\mathrm{S}=2, \mathrm{~L}=2)$, the ground state of the ion is a singlet i.e. is nonmagnetic $[8,9]$. Magnetic activity of Fe ions results from the magnetic field induced mixing of the ground singlet with the excited multiplets. Eventually FeDMS show typical Van Vleck-type magnetic behaviour [8]. Moreover $\mathrm{Fe}$ ions are coupled by $\mathrm{AF}$ exchange interaction, similarly as $\mathrm{Mn}$ ions [8]. The fundamental difference between $\mathrm{Mn}$ and Fe magnetic moments puts the question about possible SG phase formation for Fe-based DMS $[10,11]$.

In experiments performed for Fe-DMS so far, an anomaly in a.c. susceptibility was indeed observed, whereas magnetic specific heat showed no anomaly at the corresponding temperature $[10,11,12,13]$. This behaviour was ascribed to paramagnetic - SG phase transition. 
Unfortunately only limited data were collected for Fe-DMS and moreover no d.c. susceptibility history dependent experiments were performed for these materials [2]. This makes conclusions about SG formation much weaker, than for Mn-DMS. Therefore we thought it worthwhile to perform d.c. experiments, to check the third experimental evidence of SG behaviour.

We used four Fe-DMS crystals: $\mathrm{Zn}_{0.74} \mathrm{Fe}_{0.26} \mathrm{~S}$, $\mathrm{Zn}_{0.792} \mathrm{Fe}_{0.208} \mathrm{Se}, \quad \mathrm{Hg}_{0.89} \mathrm{Fe}_{0.11} \mathrm{Se}$ and $\mathrm{Cd}_{0.84} \mathrm{Fe}_{0.16} \mathrm{Se}$. The ZnFeSe sample was the same one, which was previously used for a.c. susceptibility and specific heat study [10]. We chosen crystals with possibly high Fe concentration to be able to observe the magnetic transition above $2 \mathrm{~K}$, which was our lowest experimental temperature. It follows from the previous investigation of Fe-DMS [11], that usually there are precipitations of $\mathrm{Fe}_{x} \mathrm{~S}_{y}\left(\mathrm{Fe}_{x} \mathrm{Se}_{y}\right)$ in the crystals, which provide ferromagnetic contribution to the measured susceptibility. For $\mathrm{x}<0.1$, roughly speaking about $1 \%$ of $\mathrm{Fe}$ ions precipitate in ferromagnetic contaminations [11]. For our concentration range one should expect even higher amount of precipitations, since we are close to the limit of single phase Fe-DMS crystals.

We measured d.c. magnetization of our samples using a SQUID magnetometer operating in the range 2$375 \mathrm{~K}$ and $\mathrm{B}<5.75 \mathrm{~T}$. The experimental procedure was the following:
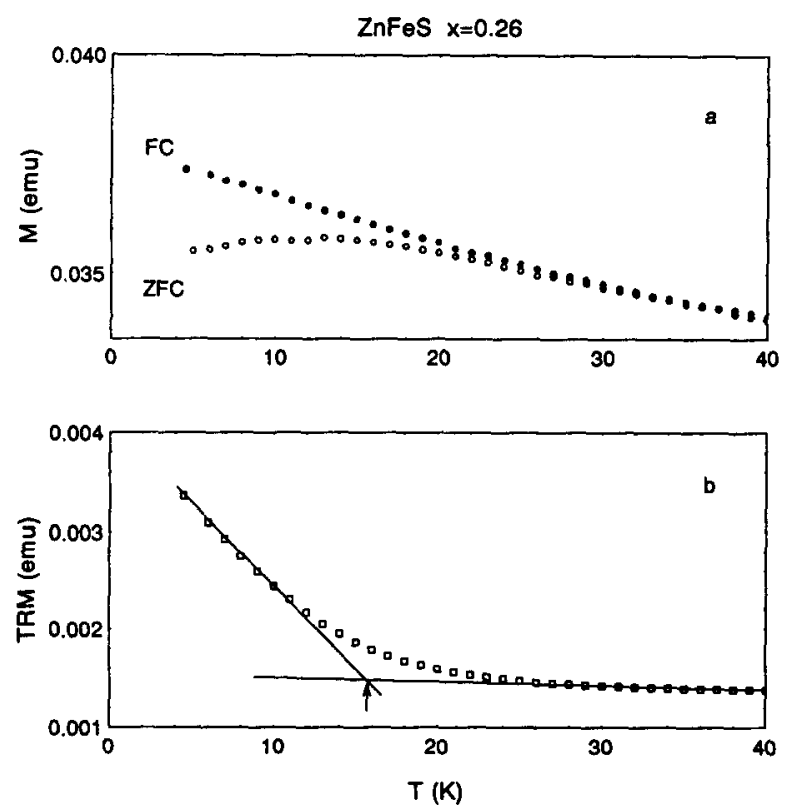

Fig.1 a). Field cooled (FC) and zero field cooled (ZFC) magnetization of $\mathrm{Hg}_{0.89} \mathrm{Fe}_{0.11} \mathrm{Se}$ as a function of temperature, taken at $\mathrm{B}=0.1 \mathrm{~T}, \mathrm{~b})$. Thermoremanent magnetization (TRM) of $\mathrm{Hg}_{0.89} \mathrm{Fe}_{0.11} \mathrm{Se}$ as a function of temperature, taken at $B=0$ after cooling at $B=0.1 T$. The diamagnetic contribution corrections (HgSe host lattice and sample holder) are negligible. Sample's mass was $0.10498 \mathrm{~g}$. The dashed lines are to guide the eye only. $T_{f}=3.8 \mathrm{~K}$.
- ZFC experiment: the sample was cooled in the absence of external magnetic field from $T=300 \mathrm{~K}$ to $\mathrm{T}=2 \mathrm{~K}$. Then the field $B=0.1 \mathrm{~T}$ was applied and magnetization of the sample was measured as a function of increasing temperature. The resulting magnetization is denoted as $\mathrm{M}_{\mathrm{zFC}}$.

- FC experiment: the sample was cooled in the presence of magnetic field $B=0.1 T$ from $T=300 K$ to $T=2 K$. Than magnetization was measured as a function of increasing temperature (magnetic field was kept at $0.1 \mathrm{~T}$ ). The resulting magnetization is denoted as $\mathbf{M}_{\mathrm{FC}}$.

- TRM experiment: the sample was cooled in the presence of magnetic field $B=0.1 \mathrm{~T}$ from $\mathrm{T}=300 \mathrm{~K}$ to $\mathrm{T}=2 \mathrm{~K}$. Then the field was suppressed to zero and magnetization was measured as a function of increasing temperature (at $\mathrm{B}=0$ ). The resulting magnetization is denoted as $M_{T R M}$.

We should notice that the residual magnetic field (i.e. the "zero" field) of our magnetometer was not higher then $0.001 \mathrm{~T}$

The obtained results are displayed in Figs.1 (HgFeSe), 2 (CdFeS), 3(ZnFeSe) and 4 (ZnFeS). We show the raw magnetization data, neither corrected for the host lattice diamagnetic contribution, nor for the sample holder, since both corrections are negligible. For all the systems, at sufficiently high temperatures a typical paramagnetic behaviour is observed: there is no difference between ZFC and FC magnetization. Below certain temperature (which
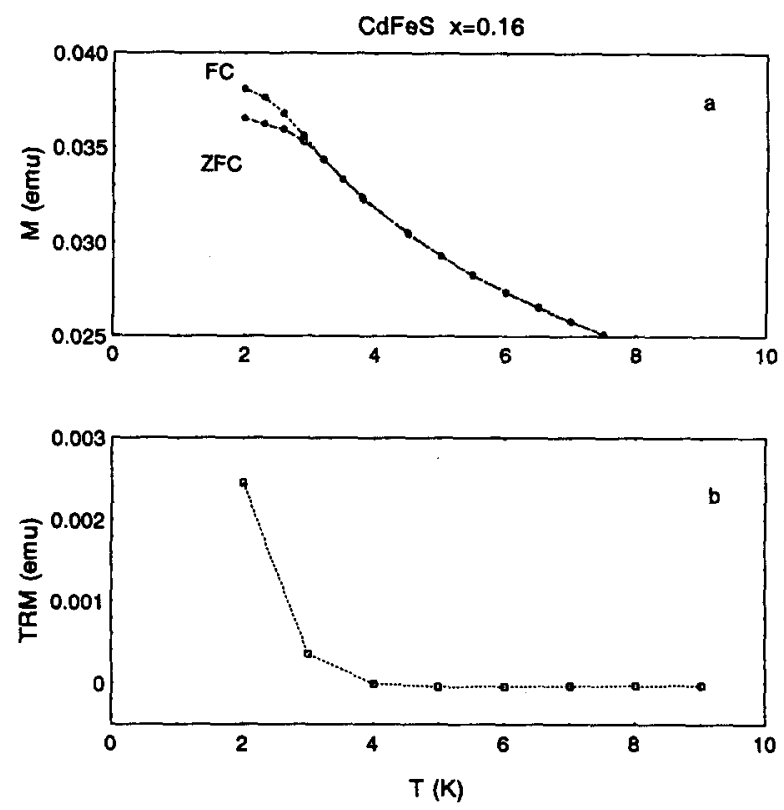

Fig.2 a). Field cooled (FC) and zero field cooled (ZFC) magnetization of $\mathrm{Cd}_{0.84} \mathrm{Fe}_{0.16} \mathrm{~S}$ as a function of temperature, taken at $\mathrm{B}=0.1 \mathrm{~T}, \mathrm{~b})$. Thermoremanent magnetization (TRM) of $\mathrm{Cd}_{0.84} \mathrm{Fe}_{0.16} \mathrm{~S}$ as a function of temperature, taken at $\mathrm{B}=0$ after cooling at $\mathrm{B}=0.1 \mathrm{~T}$. The diamagnetic contribution corrections (CdS host lattice and sample holder) are negligible. Sample's mass was $0.19998 \mathrm{~g}$. The dashed lines are to guide the eye only. $\mathrm{T}=2.9 \mathrm{~K}$. 
we denote as $\left.T_{0}\right)$, different for different systems $\left(T_{0}-4 \mathrm{~K}\right.$ for $\mathrm{HgFeSe}, 3 \mathrm{~K}$ for $\mathrm{CdFeS}, 15 \mathrm{~K}$ for $\mathrm{ZnFeSe}$ and about $20 \mathrm{~K}$ for $\mathrm{ZnFeS}$ ), ZFC magnetization starts to deviate from FC magnetization, similarly as was observed for Mn-based DMS [5,7]. Moreover nonzero TRM, increasing with decreasing temperature, is observed. For $T>T_{0} T R M$ stabilizes at a constant value, which we denote as $M_{0}$ (different for different systems).

The difference between FC and ZFC magnetization, as well as nonzero TRM is typical for a SG phase. In the ideal case of SG-paramagnetic transition one should expect total quenching of TRM at $T>T_{r}$. This is clearly not the case for $\mathrm{ZnFeS}, \mathrm{ZnFeSe}$ and $\mathrm{HgFeSe}$, for which a constant contribution to TRM $\left(M_{0}\right)$ is observed even at temperatures where $\mathrm{M}_{\mathrm{ZF} C} \approx \mathrm{M}_{\mathrm{FC}}$. This additional contribution $\left(\mathrm{M}_{0}\right)$ is too large to result from the residual magnetic field of our magnetometer $(<0.001 \mathrm{~T})$ and most probably is due to the ferromagnetic precipitations present in our crystals [11]. The ordering temperatures of the precipitations are of the order of hundred $K$ [14], which should yield approximately constant contribution in our temperature range. In order to check this hypothesis we performed the following
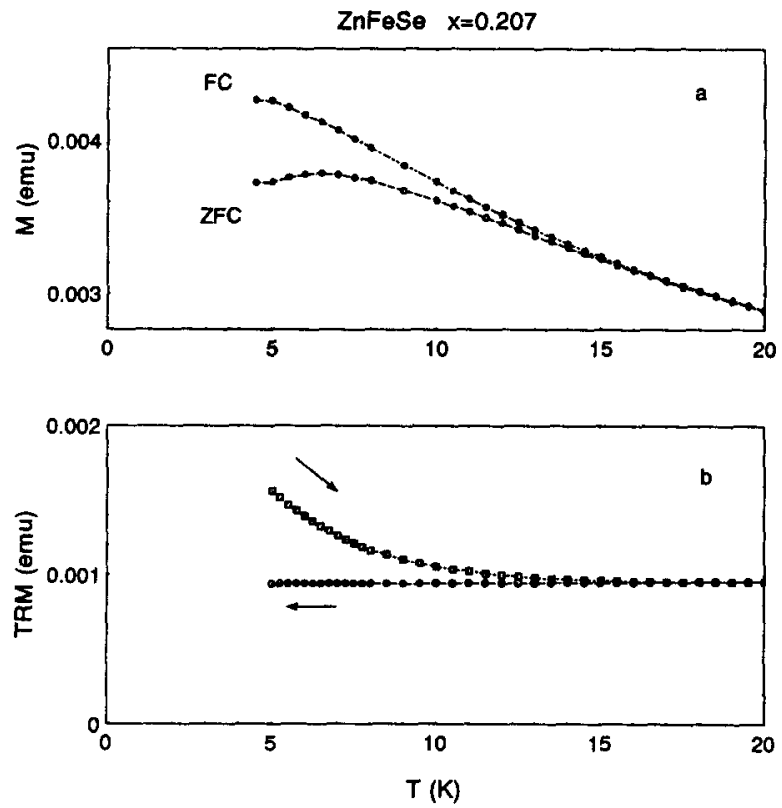

Fig.3 a). Field cooled (FC) and zero field cooled (ZFC) magnetization of $\mathrm{Zn}_{0.792} \mathrm{Fe}_{0.208} \mathrm{Se}$ as a function of temperature, taken at $\mathrm{B}=0.1 \mathrm{~T}, \mathrm{~b})$. Thermoremanent magnetization (TRM) of $\mathrm{Zn}_{0.792} \mathrm{Fe}_{0.208} \mathrm{Se}$ as a function of temperature, taken at $B=0$ after cooling at $B=0.1 T$ (upper data) and magnetization measured as a function of decreasing temperature (from $20 \mathrm{~K}$ to $5 \mathrm{~K}$ ), after cooling the sample at $B=0.1 \mathrm{~T}$, suppressing the field to zero, then warming the sample to $20 \mathrm{~K}$. The diamagnetic contribution corrections (ZnSe host lattice and sample holder) are negligible. Sample's mass was $0.05881 \mathrm{~g}$. The dashed lines are to guide the eye only. The estimated $\mathrm{T}_{r}=8.5 \mathrm{~K}$. experiment for $\mathrm{ZnFeSe}$ : TRM was measured, as described above, up to $T=20 \mathrm{~K}$, i.e. to the temperature at which $S G$ phase should be defrozen and SG-related TRM should vanish (c.f. FC and ZFC results in Fig. 3a). Next the magnetization was measured during cooling the sample in the absence of magnetic field, down to 5K. Since no magnetic field was applied, no magnetic moment (associated with SG phase transition) should be frozen and no additional magnetization should arise. The results shown in Fig. $3 \mathrm{~b}$ demonstrate that indeed the residual magnetization does not change with decreasing temperature, which means that only the difference $M_{\text {TRM }}-M_{0}$ can be ascribed to the $S G$ phase. This conjecture is additionally corroborated by the observation that $\mathrm{M}_{\mathrm{FC}}(T)-\mathrm{M}_{\mathrm{ZFC}}(\mathrm{T}) \approx \mathrm{M}_{\mathrm{TRM}}(T)-\mathrm{M}_{0}$.

We note that for $\mathrm{HgFeSe}$ and CdFeS the transition is relatively sharp, and therefore $T_{t}$ is relatively well defined $\left(T_{r}=3.8 \mathrm{~K}\right.$ for $\mathrm{HgFeSe}$ and $\mathrm{T}_{\boldsymbol{r}}=2.9 \mathrm{~K}$ for $\left.\mathrm{CdFeS}\right)$. On the other hand for both $\mathrm{ZnFeSe}$ and $\mathrm{ZnFeS}$ the transition is quite broad and it's rather difficult to define $T_{r}$. The broadness of the transition seems to be related to the presence of ferromagnetic contaminations: it is the strongest for $\mathrm{ZnFeSe}$, for which the precipitations-induced magnetization $\mathbf{M}_{0}$ is also the largest. In such a case we estimated $T_{f}$ from the intersection of the extrapolated TRM
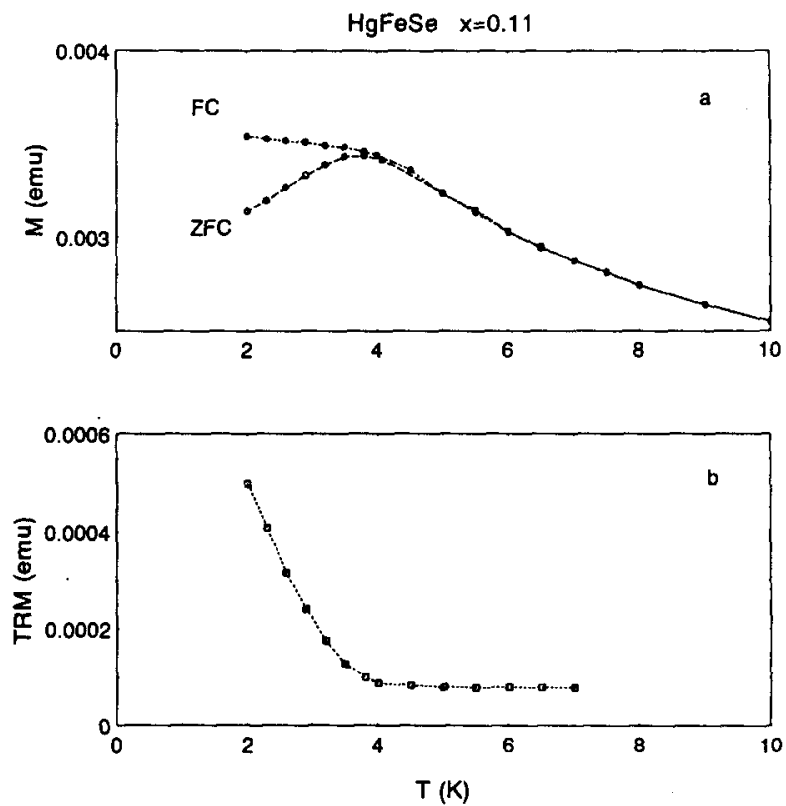

Fig.4 a). Field cooled (FC) and zero field cooled (ZFC) magnetization of $\mathrm{Zn}_{0.74} \mathrm{Fe}_{0.26} \mathrm{~S}$ as a function of temperature, taken at $B=0.1 \mathrm{~T}, \mathrm{~b})$. Thermoremanent magnetization (TRM) of $\mathrm{Zn}_{0.74} \mathrm{Fe}_{0.26} \mathrm{~S}$ as a function of temperature, taken at $B=0$ after cooling at $B=0.1 \quad T$. The diamagnetic contribution corrections ( $\mathrm{ZnS}$ host lattice and sample holder) are negligible. Sample's mass was $0.15334 \mathrm{~g}$. The arrow show the estimate for $T_{t}=15.7 \mathrm{~K}$. 
magnetization well below and well above the transition (Fig.4b), obtaining $T_{r}=8.5 \mathrm{~K}$ for $\mathrm{ZnFeSe}$ and $T_{r}=15.7 \mathrm{~K}$ for $\mathrm{ZnFeS}$.

The obtained $T_{f}$ values are plotted versus $x$ in Fig.5, together with the other available data $[12,17,18]$. For $\mathrm{Mn}$ DMS the SG phase transition was analyzed on the grounds of scaling analysis $[4,6,7]$. Such an analysis generally exploits the fact that for a continuous, random distribution of magnetic ions the product $\mathrm{xR}_{\mathrm{ij}}{ }^{3}$ should be constant, where $R_{i j}$ is typical distance between the ions. Implementing this expression in a model of a SG freezing, given a known form of exchange interaction distance dependence, then yields a prediction for $T_{\mathbb{R}}(x)$. For all Mn-DMS $T_{\mathbf{f}}$ was found to obey the relation [2]:

$$
\ln T_{f} \propto \frac{n}{3} \ln x
$$

which is compatible with a radial dependence of exchange interaction $J(R) \propto R^{-n}$, where $n=6.8$ for wide gap materials (such as CdMnTe, ZnMnSe) and $\mathrm{n}=5$ for narrow gap crystals (HgMnTe, $\mathrm{HgMnSe}$ ) [2,7]. The longer range of exchange for the latter was attributed to BlombergenRowland-type exchange, particularly effective in the case of a narrow gap semiconductor. Following the scaling analysis we find that also for Fe-DMS relation (1) seems to hold (Fig.5). The wide gap systems (ZnFeS, ZnFeSe, CdFeS, $\mathrm{CdFeSe}$ ) reveal a common behaviour corresponding to eq. 1 with $n=10.8$. Such a large $n$ value must not necessarily mean the shorter interaction range for Fe-DMS, as pointed in Ref.10. We recall that for magnetic ions with a singlet ground state the real interaction distance dependence may be masked by the ground state splitting and then $T_{N}(x)$ may not uncover $J(R)$ dependence. However if we ascribe the large value of $n$ to the particular energy structure of $F e$ ion, one should expect similar effects for Fe-based narrow gap DMS (HgFeSe, HgCdFeSe). Apparently this is not the case, since for these crystals we find $n=5.0$ (Fig.5), which is the same as for the corresponding Mn-based DMS. Therefore it seems that no pertinent conclusions concerning exchange interaction range for Fe-DMS can be derived without developing a precise model of freezing of $\mathrm{Fe}^{++}$ion system.

Summarizing our results we notice that for Fe-based DMS we observe the same type of magnetic behaviour which was reported for Mn-based DMS and was ascribed to paramagnetic - SG phase transition [2]. This may suggest that field induced magnetic moments of $\mathrm{Fe}$ ions can be

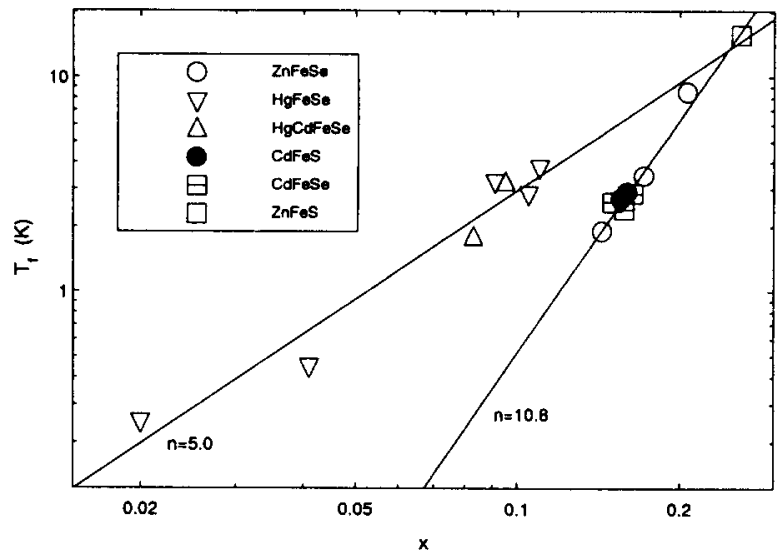

Fig.5 Magnetic phase diagram (freezing temperature versus $\mathrm{Fe}$ concentration) for Fe-Diluted Magnetic Semiconductors resulting from d.c. magnetometry [this paper] and a.c. susceptibility $[12,13,17,18]$. The lines indicate the dependence $\ln T_{f} \propto(n / 3) \ln x$.

frozen into spin-glass-like phase. Since no microscopic mechanism of the freezing was proposed so far $[1,2]$ we think that some other experiments, like frequency dependence of magnetization or neutron diffraction, providing information about correlation range between $\mathrm{Fe}$ magnetic moments, would be useful in deriving final conclusions about the SG phase for Fe-DMS. Moreover the phase diagram (freezing temperature versus $\mathrm{Fe}$ concentration) for these materials should be established in a broader concentration range than presented here. This however will be difficult unless crystals with higher $\mathrm{Fe}$ concentration are available [2]. Hopefully epitaxial growth techniques will be useful in this task [15].

Acknowledgments: We acknowledge the support from National Science Council and Physics Center grant no. NSC-84-2912-I-033-153-A2 (Taiwan) and Committee for Scientific Research (Poland)

References:

1. Semiconductors and Semimetals, 25, "Diluted Magnetic Semiconductors" ed. J.K.Furdyna and J.Kossut, Academic Press (1988)

"Diluted Magnetic Semiconductors", ed. M.Balkanski and M.Averous, Plenum Press (1991) J.Kossut and W.Dobrowolski, in Handbook of Magnetic Materials, ed. K.H.J.Buschow, 7, 231 (1993), North Holland, Amsterdam
2. A.Twardowski, Physica Scripta, T39, 124 (1991) W.J.M de Jonge and H.J.M.Swagten, J. Magnetism and Magn.Mat. 100, 322 (1991)

3. see review papers: J.A.Mydosh in "Hyperfine Interactions", vol. 31 of Lecture Notes in Physics, p.347 (Springer, New York 1986)

K.Binder and A.P.Young, Rev. Mod. Phys. 56, 801 (1986) 
4. Except of II-V DMS, for which the host lattice is a high symmetry one. See: C.J.M.Denissen, H.Nishihara, J.C. van Gool and W.J.M. de Jonge, Phys. Rev. B33, 7637 (1986)

C.J.M.Denissen, Sun Dakun, K.Kopinga and W.J.M. de Jonge, Phys. Rev. B36, 5316 (1987)

5. R.R.Gałązka, S.Nagata and P.H.Keesom, Phys. Rev. B22, 3344 (1980)

6. M.A.Novak, O.G.Symko, D.J.Zheng and S.Oseroff, J. Appl. Phys. 57, 3418 (1985)

7. A.Twardowski, H.J.M.Swagten, W.J.M. de Jonge and M.Demianiuk, Phys.Rev. B36, 7013 (1987)

8. A.Twardowski, J.Appl. Phys. 67, 5108 (1990), A.Twardowski in "Diluted Magnetic Semiconductors", ed. M.Balkanski and M.Averous, Plenum Press (1991)

9. W.Low and M.Weger, Phys.Rev. 118, 1119 (1960) G.A.Slack, S.Roberts and J.T.Vallin, Phys.Rev. 187, $511(1969)$
10. H.J.M.Swagten, A.Twardowski, W.J.M.de Jonge and M.Demianiuk, Phys. Rev. B39, 2568 (1989)

11. A.Twardowski, H.J.M.Swagten, W.J.M.de Jonge and M.Demianiuk, Phys. Rev. B44, 2220 (1991)

12. M.Arciszewska, A.Lenard, T.Dietl, W.Plesiewicz, T.Skośkiewicz and W.Dobrowolski, Acta Physica Polonica, A77, 155 (1990)

13. A.Lewicki, J.Spałek and A.Mycielski, J.Phys. C, 20, 2005 (1987)

14. Landoldt-Bornstein, Il/12a (Springer, 1984)

15. B.T.Jonker, J.J.Krebs, S.B.Quadri and G.A.Prinz, Appl. Phys. Lett. 50, 848 (1987)

16. S.Geshwind, D.A.Huse and G.E.Devlin, J. Appl. Phys. 67, 5249 (1990)

17. M.Arciszewska, $\mathrm{PhD}$ Thesis, Institute of Physics, Polish Academy of Sciences (1993), unpublished

18. A.Lenard, $\mathrm{PhD}$ Thesis, Institute of Physics, Polish Academy of Sciences (1993), unpublished 